\title{
Novel Spatial Modulation Channel Index Detection in Optical Wireless Communications with Signal Space Diversity
}

\author{
Tingting Song \\ Department of Electrical and Electronic Engineering \\ The University of Melbourne \\ Parkville, VIC 3010, Australia \\ tingtings1@student.unimelb.edu.au \\ Ampalavanapillai Nirmalathas \\ Department of Electrical and Electronic Engineering \\ The University of Melbourne \\ Parkville, VIC 3010, Australia \\ nirmalat@unimelb.edu.au \\ Christina Lim \\ Department of Electrical and Electronic Engineering \\ The University of Melbourne \\ Parkville, VIC 3010, Australia \\ chrislim@unimelb.edu.au
}

\author{
Elaine Wong \\ Department of Electrical and Electronic Engineering \\ The University of Melbourne \\ Parkville, VIC 3010, Australia \\ ewon@unimelb.edu.au \\ Kamal Alameh \\ Electron Science Research Institute (ESRI) \\ Edith Cowan University \\ Joondalup, WA 6027, Australia \\ k.alameh@ecu.edu.au \\ Ke Wang \\ School of Engineering \\ RMIT University \\ Melbourne, VIC 3000, Australia \\ ke.wang@rmit.edu.au
}

\begin{abstract}
A novel spatial modulation channel index detection algorithm using a joint maximum-a-posteriori estimation is proposed for optical wireless communications with 4-CAP modulation and transmitter diversity. Diversity gain, enabling improvement of about $0.4 \mathrm{~dB}$ in SNR and 1.5-times in data rate, is achieved with reduced computation complexity.
\end{abstract}

Keywords—Channel index, optical wireless communications, signal space diversity, spatial modulation

\section{INTRODUCTION}

The increasing demands for remote working/education and high definition video streaming pose a significant challenge to current indoor wireless networks. Apart from the radio frequency (RF) wireless communication, line-of-sight optical wireless communication (LoS-OWC) is a promising solution [1], since it can achieve high-capacity connections with competitive advantages of scalable bandwidth, simple deployment leveraging the fiber-to-the-premise/home broadband networks, and immunity against RF interference.

One of the critical challenges in high-capacity LoS-OWC systems, which is the optical beam blocking, can be addressed by providing redundant spatial links using spatial diversity. To further increase the data rate and take advantage of the channel gain difference caused by the partial beam blocking simultaneously, the spatial modulation (SM) technique with transmitter diversity using signal space diversity (SSD) has been proposed $[2,3]$. In this scheme, data from each transmitter was modulated using 4Carrierless Amplitude and Phase (CAP) modulation. However, in previous studies [3, 4], the case of wrong SM channel recognition was never investigated, since the main focus was laid on the performance of SSD. In this paper, we propose a simplified 4-CAP modulation implementation using only one pair of I/Q filters and a practical novel SM channel index detection algorithm using a joint maximum-a-posteriori (MAP) estimation at the receiver. Compared with local maximum-likelihood (ML) estimation using the same adjacent data range factor $\tau$, the proposed joint MAP estimation can provide a $2 / 3$ less computation complexity with a slightly better bit-error-rate (BER) performance. Besides, the proposed approach can achieve a diversity gain, which enables $0.4 \mathrm{~dB}$ signalto-noise ratio (SNR) improvement at $45^{\circ} \mathrm{SSD}$ rotation angle.

\section{PRINCIPLES}

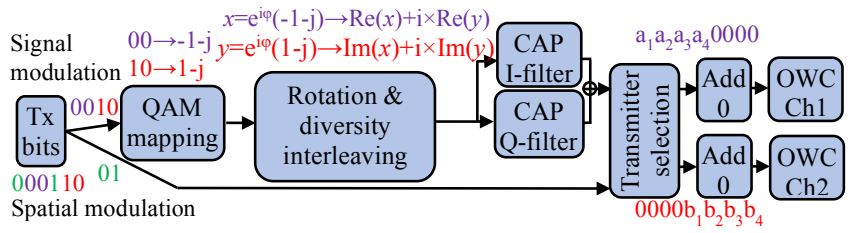

(a)

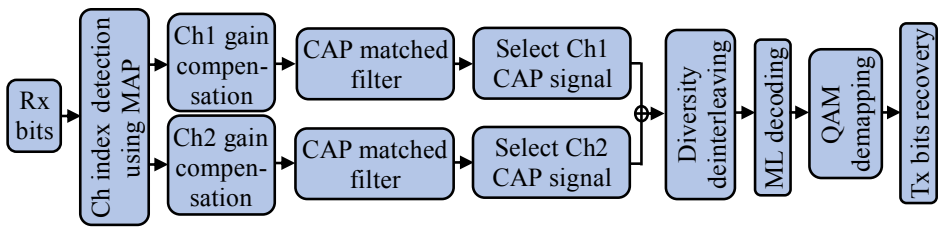

(b)

Tx - Transmitter; Ch1, Ch2 - Channel 1, Channel 2; Rx - Receiver; ML - Maximum Likelihood

Fig. 1. Block diagram of SM using novel channel index detection algorithm along with SSD (a) Transmitter; (b) Receiver (after photodetection). 
For the signal coding at the transmitter, SSD is performed with signal rotation followed by diversity interleaving, as shown in Fig.1 (a). Then the signal for both transmitters is 4-CAP modulated using only one pair of CAP filters before the SM transmitter selection, which is simplified compared to that in [3] and enables lower complexity implementation. For the signal detection at the receiver, 4-CAP training symbols are used to obtain the 4-CAP signal value distribution, as illustrated in Fig. 2, with a signal value precision rounded to 2 decimal places to reasonably reduce the possible $x_{j}(t)$ values. Then the channel index $\hat{I}(t)$ is estimated using a joint MAP [2], shown in (1). Note that the conditional probability in (1) is also obtained through training.

$$
\hat{I}(t)=\arg \max _{I \in\{1,2\}, x_{j}^{I}(t) \in x^{I}(t)}\left(\sigma^{2}\left(\ln P_{x_{1}^{I}(t)}+\ln P_{\left(x_{2}^{I}(t) \mid x_{1}^{I}(t)\right)}+\ln P_{\left(x_{3}^{I}(t) \mid x_{2}^{I}(t)\right)}+\ln P\left(x_{4}^{I}(t) \mid x_{3}^{I}(t)\right)\right)-\left\|\boldsymbol{y}-h_{I} \boldsymbol{x}^{I}(\boldsymbol{t})\right\|^{2}\right)
$$

where $\sigma$ is the noise variance, $P_{x_{1}^{I}(t)}$ is the probability of $x_{1}^{I}(t), P_{\left(x_{m}^{I}(t) \mid x_{n}^{I}(t)\right)}$ is the conditional probability of $x_{m}^{I}(t)$ given $x_{n}^{I}(t)$ with $(\mathrm{m}, \mathrm{n}) \in(2,1),(3,2)$ or $(4,3), \boldsymbol{y}$ is the received electrical signal vector, $h_{I}$ is the corresponding channel gain, $\boldsymbol{x}^{I}(\boldsymbol{t})$ is the possible transmitted signal vector with $\boldsymbol{x}^{I}(\boldsymbol{t})=\left\{x_{j}^{I}(t) \in\left[y_{j} / h_{I}-\tau, y_{j} / h_{I}+\tau\right], j=1,2,3,4\right\}$ and $\tau$ is the adjacent data range factor.

The estimated channel index is then used for the corresponding channel gain compensation, as is shown in Fig.1 (b). To ensure the accuracy of subsequent CAP signal demodulation, the channel gain compensation is performed separately for each channel, with channel 1's gain compensated to that of channel 2, and vice versa. The compensated signals then go through the CAP matched filter separately for demodulation, and the two output signals are recombined according to their corresponding channel selection in SM. The subsequent processes, such as diversity deinterleaving and ML decoding, are similar to that described in [3].

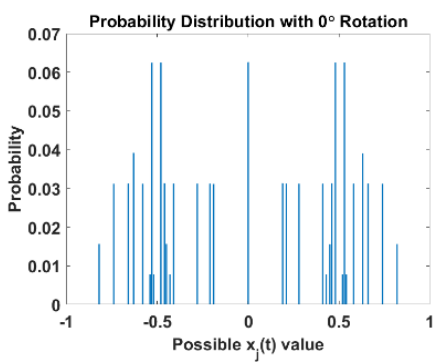

(a)

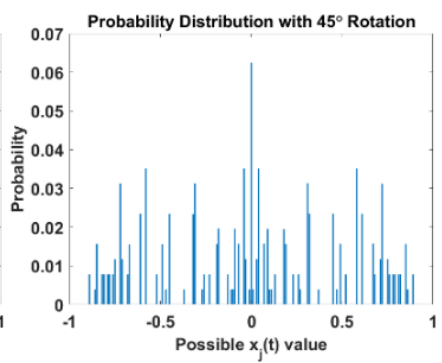

(b)

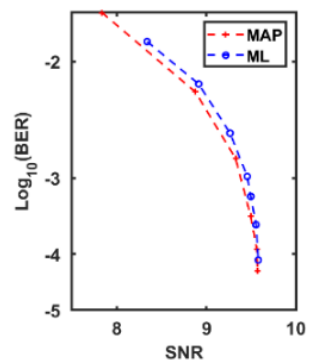

(a)

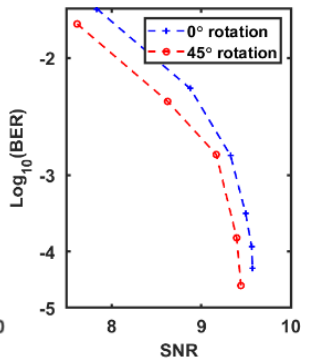

(b)

Fig. 2. Probability distribution of $x_{j}(t)$ value with SSD rotation (a) $0^{\circ}$; (b) $45^{\circ}$. Fig. 3. Numerical results (a) MAP vs. ML estimation. (b) SSD rotation: $0^{\circ}$ vs. $45^{\circ}$.

\section{NUMERICAL SIMULATIONS}

Numerical simulation results of the system BER performance using the proposed joint MAP estimation and using the local ML estimation with $0^{\circ} \mathrm{SSD}$ rotation angle is compared in Fig. 3 (a). The local ML estimation is conducted using the same adjacent data range factor $\tau$ as that for the joint MAP estimation. Using $\tau$ for local ML estimation is reasonable, since $\tau$ is larger than the maximum channel noise, and the transmitted signal is ensured to falls in the $\tau$ range. It can be seen that the joint MAP estimation results in a slightly better BER performance. Besides, the computation complexity of joint MAP estimation is much lower than that of the local ML estimation since fewer $x_{j}(t)$ symbols have valid values with the probability distribution shown in Fig. 2 . This in turn reduces the number of computations by about $2 / 3$ through the simulation where $\tau=0.17$ and SNR $=9.45$.

Numerical simulation results of the system BER performance with $0^{\circ}$ and $45^{\circ} \mathrm{SSD}$ rotation angles using the proposed joint MAP estimation is shown in Fig. 3 (b). $45^{\circ}$ rotation angle is selected for SSD due to its best diversity gain with optimum minimum constellation distance. It can be seen that SSD in the $45^{\circ}$ rotation angle provides a diversity gain that enables about $0.4 \mathrm{~dB}$ SNR improvement. Compared with the ideal channel index detection of SM [3] where SSD rotation could result in a larger SNR improvement, it can be seen that the performance of channel index detection affects the SSD diversity gain. Nevertheless, our proposed approach can efficiently detect the SM channel index and achieve diversity gain, thus addressing the challenge that CAP signals have numerous signal power levels (values) and the SM channel index detection using channel gain is typically problematic.

\section{CONCLUSIONS}

A novel efficient SM channel index detection algorithm using a joint MAP estimation has been designed for indoor OWC systems, with SSD for realizing transmitter diversity, and SM for increasing the data rate. Results have shown that, compared with the local ML estimation counterpart, the joint MAP estimation can be achieved with 2/3 less computation complexity, and competitive performance can also be attained. In addition, results have shown that with the diversity gain, an SNR improvement of about $0.4 \mathrm{~dB}$ can be achieved using the proposed channel index detection algorithm.

\section{REFERENCES}

[1] T. Koonen, "Indoor optical wireless systems: technology, trends, and applications," Journal of Lightwave Technology, vol. 36, no. 8, pp. 1459-1467, 2018.

[2] J. G. Proakis and M. Salehi, Digital communications. McGraw-hill New York, 2001.

[3] T. Song, K. Wang, A. Nirmalathas, C. Lim, E. Wong and K. Alameh, "Demonstration of Optical Wireless Communications Using Spatial Modulation with Signal Space Diversity," in IEEE Photonics Conference, 2019, pp. WB1.3.

[4] S. Althunibat and R. Mesleh, "Enhancing spatial modulation system performance through signal space diversity," IEEE Communications Letters, vol. 22, no. 6, pp. 1136-1139, 2018.

This work was supported by the Australian Research Council Discovery Project DP170100268. 


\section{University Library}

\section{- M M N E R VA A gateway to Melbourne's research publications}

Minerva Access is the Institutional Repository of The University of Melbourne

Author/s:

Song, T;Wong, E;Nirmalathas, A;Alameh, K;Lim, C;Wang, K

Title:

Novel Spatial Modulation Channel Index Detection in Optical Wireless Communications with Signal Space Diversity

Date:

2020-01-01

Citation:

Song, T., Wong, E., Nirmalathas, A., Alameh, K., Lim, C. \& Wang, K. (2020). Novel Spatial Modulation Channel Index Detection in Optical Wireless Communications with Signal Space Diversity. 2020 IEEE PHOTONICS CONFERENCE (IPC), 00, IEEE. https://doi.org/10.1109/ IPC47351.2020.9252214.

Persistent Link:

http://hdl.handle.net/11343/258812 